\title{
Anterior Inferior Frontal Convolution
}

National Cancer Institute

\section{Source}

National Cancer Institute. Anterior Inferior Frontal Convolution. NCI Thesaurus. Code C32092.

The orbital part of the inferior frontal convolution. 